\title{
Why Continuem Damage is Nonlocal: Micromechanics Arguments
}

By Zdeněk P. Bažant,' Fellow, ASCE

ABStRact: The paper presents two micromechanics arguments showing that continuum damage caused by microcracking ought to be nonlocal, defined by at spatial integral. Argument $t$ is an analysis of a simplified model in which the microcracks are of such size, density, and arrangement that that they do not interact. The release of stored energy caused by the formation of one microcrack is calculated as a function of the associated relative displacement across the cell, which corresponds to the average strain of the macrosepic continuum. After imposing two homogto the average strain of the macroscopic continum. After imposing two homog-
enizing conditions, it is shown that damage is a nonlocal variable that is a function of the averaged (nonlocal) strain trom a cerain neighborhood of the given point Argument II is an analysis of a body with arbitrary interacting cracks. The local dimage is proportional to the forces applied on the cracks to replace the stresses before cracking. Crack formation changes the openings of the neighboring cracks, which represents an interaction described by crack influence cosfficients. The nonlocality is a consequence of crack interactions. and the weight tunction for nonlocal spatial integration appears to be related to the influence coefficients.

\section{INTRODUCTION}

In the broad sense, a nonlocal continuum is a continuum in which some variables in the constitutive equation for a point of the continuum are related through spatial averaging integrals to the field of these or other variables in a certain neighborhood of the point. Such concepts were developed in the works of Eringen $(1965,1966)$ (who originatly called it the concept of attenuating neighborhood), Kröner (1968), Krumhansl (1968), Kunin (1968), Levin (1971), Eringen and Edelen (1972), Eringen and Ari (1983), and others. Recently, the nonlocal concept was applied to structures with strain-softening continuum damage [see Bažant (1986)] and was shown effective for overcoming the numerical and stability problems plaguing the classical (local) constitutive models with strain softening (Pijaudier-Cabot and Bažant 1987; Bažant and Pijaudier-Cabot 1988; Bažant and Lin 1988a,b; Bažant and Ožbolt, 1990). The basic idea of this nonlocal continuum model is that only the strain-softening damage is nonlocal, while all the other variables, especially the elastic strain and the total strain used as a kinematic variable defining the work of stress, ought to be local.

By contrast, in the original nonlocal continuum models (which were not intended to cope with strain softening), as well as in the first nonlocal model for strain-softening continuum (Bažant 1984; Bažant ct al. 1984), the elastic strain and total strain were nonlocal. This led to certain numerical difficultics (Bažant and Pijaudier-Cabot 1988), particularly the existence of spurious zeroenergy instability modes (which had to be suppressed artificially by overlay with local continuum), the presence of spatial integrals or higher-order derivatives in the differential equations of equilibrium or motion and in the boundary and interface conditions, and an imbricate structure of the finite

Walter P. Murphy Prof. of Civ. Engrg., Northwestern Univ., Evanston, IL 60208. Note. Discussion open until October 1, 1991. To extend the closing date one month, a written request must be filed with the ASCE Manager of Journals. The manuscript for this paper was submitted for review and possible publication on July 12, 1990. This paper is part of the Journal of Engineering Mechanics, Vol. 117, No. 5, May, 1991. OASCE, ISSN 0733-9399/91/0005-1070/\$1.00+\$.15 per page. Paper No. 25803. 
element approximation that proved cumbersome for programming. These difficulties were later shown to be a consequence of imposing symmetry on the integral or differential operators involved. The symmetry is lost with the nonlocal damage concept, which means that the tangential (but not the elas(ic) structural stiffness matrix of the finite element approximation is nonsymmetric (Bažant and Pijaudier-Cabot 1988). But this does not seem to cause any serious numerical difficulties (Bažant and Ožbolt 1990), even for strain-softening structures with thousands of nodal displacements (Bažant and Lin 1988a).

The nonlocal concept eliminates problems of spurious mesh sensitivity and incorrect convergence. It ensures that refinements of finite element mesh cannot lead to spurious localization of strain, damage, and energy dissipation into a strain-softening zone of vanishing volume. The most important physical property of nonlocal continuum damage is that, for geometrically similar structures, it yields a size effect that is transitional between plasticity (no size effect) and linear elastic fracture mechanics (the strongest possible size effect). This size effect is cvidenced by extensive laboratory measurements on various kinds of concrete structures and fracture specimens of concrete and rock, as well as the avalable test data for fracture of ice le.g., Dempsey $(1990)]$ and toughening coramics [see Bažant and Kazemi (1990) with further references]. The finite element codes based on local continuum cannot capture the size effect, which is a major fault when structures with damage are analyzed. But the codes with nonlocal damage give the correct size effect, in agreement with tests (Bažant and Ožbolt 1990; Ožbolt and Eligehausen 1990). Correct modeling of the experimentally observed size effect should be adopted as the basic criterion of acceptability of any finite element code for concrete structures, rock masses, or sea ice plates.

Physical justification by micromechanics, however, has been rather limited. In a recent study (Bažant 1987), it was suggested that the physical source of nonlocality of damage is the fact that the formation and growth of a microcrack depends on the strain energy stored in a nonzero volume of the material surrounding the microcrack, whose release drives the growth of the microcrack. Considering a quasi-periodic microcrack array and analyzing the displacements caused by fracture. it was shown that, under certain simplifying assumptions, the damage is a function of the spatially averaged fracturing strain of the macroscopic smoothing continuum, which implies that damage is nonlocal. This form of damage, however, does not seem to be the most convenient formulation, and does not quite agree with the nonlocal damage formulations used in the aforementioned finite element models.

The purpose of this paper is to present two different micromechanics arguments, each showing that, indeed. continuum damage ought to be a nonlocal variable. The first argument is a modification and improvement of the argument offered in Bažant (1987), which was based on homogenization of the deformations caused by cracking in an elastic solid with a quasi-periodic array of noninteracting small microcracks. The second argument deals with the consequences of interactions among microcracks. Furthermore, there is another argument based on the effect of microstructural inhomogeneities, which seems, however, to defy simple analytical treatment and will not be presented in detail because it has been studied elsewhere numerically.

Damage caused by physical phenomena other than microcracking will not be considered.

\section{Argument I. Energy Release by Microcrack}

Consider an elastic material with penny-shaped microcracks of various diameters $2 a$. We imagine the material to be subdivided into cubical cells of side $\ell[$ Fig. $\mid(a)]$, each of which contains one microcrack approximately in the middle. For the sake of simplicity, we suppose each microcrack to bo so small $(a \& b)$ that its interaction with other microcracks, as wcll as the energy release from the adjacent cells, is negligible. We analyze one microcrack and align the cell so that its one side, as well as the coordinate axis $y$, will be parallel to this microcrack [Figs. $|(a),(b)|$. We assume that the microcrack plane is normal to the maximum principal stress at the center of the cell before cracking, denoted as $\sigma$, and, for the sake of simplicity, wo assume that the normal strains in the direction parallel to the crack are constant, as illustrated by imagined sliding restraints on the sides of the cell shown in Fig. $1(b)$. We assume the variation of $\sigma$ over the cell to be sufficiently small, so that the stress intensity factor $K_{I}$ of the microcrack is approximately the same as that for a penny-shaped crack in an infinite elastic solid with stress $\sigma$ at infinity, which is as follows [see Broek (1974), Knott (1973), Tada et al. (1985), and Murakami (1987)]

$K_{l}=2 \sigma \sqrt{\frac{a}{\pi}}$

We now try to calculate the energy release resulting from crack formation as a function of the deformation of the cell. We begin by writing the rate of release of energy (complementary energy) $W_{j}^{*}$ resulting from fracture

$\frac{\partial W_{f}^{*}}{\partial a}=2 \pi a \frac{K_{l}^{2}}{E^{\prime}}=\frac{8 \sigma^{2} a^{2}}{E^{\prime}}$.

where $E^{\prime}=E /\left(1-v^{2}\right) ; E=$ Young's elastic modulus; $v=$ Poisson's ratio. Since the material is elastic and thus path-independent, we can consider, for the purpose of energy calculation, that the crack has formed under constan stress $\sigma$. Then, by integration of Eq. 2, the total energy release caused by the microcrack is obtained as

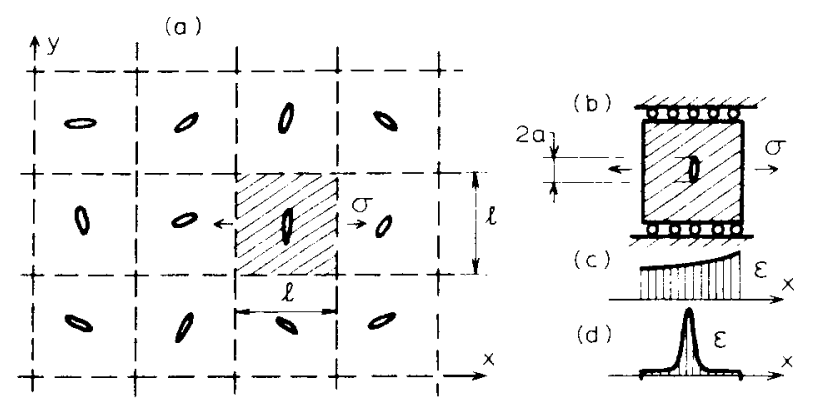

FIG. 1. (a) Array of Cubical Cells Containing Microcracks; and (b) One Cell with Simplified Boundary Conditions Considered in Calculations 

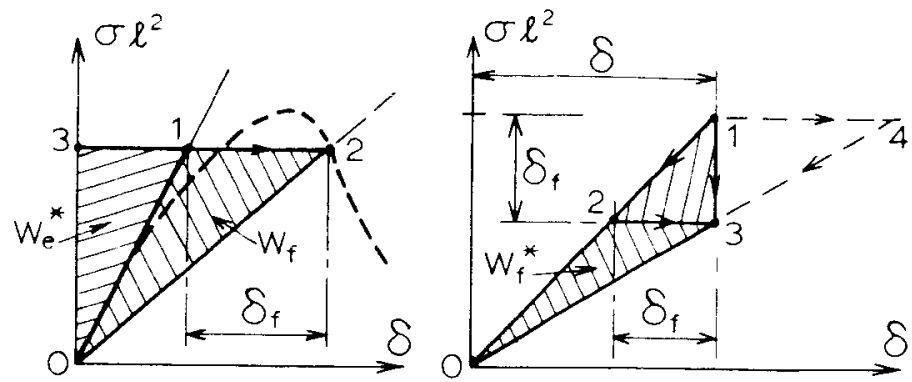

FIG. 2. Energy Released due to Crack Formation: (a) At Constant Stress; and (b) At Constant Displacement

$W_{i}^{*}=\frac{8 a^{3} \sigma^{2}}{3 E^{\prime}}$

Let $\delta$ be the total relative displacement between the opposite sides of the cell $\left(\delta=u_{2}-u_{1}\right.$ where $u_{2}, u_{1}=$ displacements in the $x$-direction at the opposite sides of the cell) and $\delta$, the relative displacement caused by crack formation, which is approximately equal to the relative displacement in an infinite solid between its opposite infinities. In the diagram of $\sigma \ell^{2}$ (the force acting on the sides of the cell) versus $\delta[\mathrm{Fig} .2(a)], W^{*}$ is represented by the area 0120. This triangular area is equal to $W_{f}^{*}=\delta_{f}\left(\sigma \ell^{2} / 2\right.$. Setting this equal to Eq. 3, one gets

$\delta_{f}=\frac{16}{3 E^{\prime}} \frac{a^{3}}{\ell^{2}} \sigma$

The same expression can be obtained from Eq. 3 by Castigliano's theorem. which implies that $\delta_{f}=\partial W_{t}^{*} / \partial\left(\sigma \ell^{2}\right)$.

Consider now that the crack forms at constant $\delta$ rather than at constant $\sigma$. This must be equivalent to first unloading the uncracked solid from initial stress $\sigma_{11}$ to a certain stress $\sigma_{0}-\sigma_{f}$ [path $\overline{12}$ in Fig. $\left.2(b)\right]$ and, second, letting the crack grow at constant stress [path $\overline{23}$ in Fig. $2(b)$ ], provided that $\sigma_{f}=$ $E^{\prime} \delta_{f} / \ell$ [from triangle 123 in Fig. $2(b)$ ]. The last condition guarantees that the displacement increase $\delta$, caused by crack formation at constant stress $\sigma_{11}$ $\sigma_{f}$ restores the original total displacement $\delta$ [point 3 in Fig. $\left.2(b)\right]$. In analogy to Eq. 4. we have for this process $\delta_{f}=16\left(a^{3} / \ell^{2}\right)\left(\sigma_{i j}-\sigma_{f}\right) / 3 E^{\prime}$ (segment $\overline{23}$ in Fig. 2). Since $\sigma_{f}=E^{\prime} \delta_{f} / \ell$, we get

$$
\sigma_{i}=\frac{\sigma_{0}}{1+\frac{3}{16}\left(\begin{array}{l}
t \\
a
\end{array}\right)}
$$

where $\sigma_{0}=E^{\prime} \delta / \ell=$ initial stress before cracking. The (complementary) energy released by crack formation at constant stress at $\sigma_{0}-\sigma_{f}$ is repre sented by area 0230 in Fig. $2(b)$, and the energy (not complementary energy) released by crack formation at constant displacement $\delta$ is represented by area 0130 in Fig. $2(b)$, the value of which is

$$
W_{f}=\sigma_{f} \frac{\delta}{\ell} \frac{\ell^{3}}{2}=\frac{E^{\prime} \ell \delta^{2}}{2+\frac{3}{8}\left(\frac{\ell}{a}\right)^{3}}
$$

Note that since the material is elastic, that is, path-independent, the same result must be obtained if one considers the path 143 (instead of 123) in Fig. $2(b)$, for which, first, the crack is formed at constant stress $\sigma_{0}$, and second, the cell is unloaded to restore the original relative displacement $\delta$.

For a propagating crack. we must have $K_{l}=K_{R}(a)=$ given $R$-curve (crack resistance curve) $=$ critical stress intensity factor required for further crack growth, which must be determined in advance. Eq. I with $\sigma$ replaced by $\sigma_{0}$

$\sigma_{f}$ Ipath $\overline{23}$ in Fig. $2(b)$ I then provides

$a=\frac{\pi K_{R}^{2}(a)}{4\left(\sigma_{0}-\sigma_{i}\right)^{2}}$

Substituting $\sigma_{0}=E^{\prime} \delta / t$ and Eq. 5 for $\sigma_{f}$, and solving the resulting equation for $\delta / \ell$, we acquire the relation

$\frac{\delta}{\ell}=\frac{1}{2 E^{\prime}} \sqrt{\frac{\pi}{\ell} \frac{\ell}{a}} K_{R}\left(\ell \frac{a}{\ell}\right)\left[1+\frac{16}{3}\left(\frac{a}{\ell}\right)^{3}\right]=\phi\left(\frac{\ell}{a}\right)$

where $\phi$ is the function defined by this relation. Denoting the inverse function as $\psi$ (and supposing function $\phi$ to be invertible), we can write

$\frac{\ell}{a}=\psi\left(\frac{\delta}{\ell}\right)$

Substituting this into Eq. 6, we obtain the result

$W_{1}=E^{\prime} t^{3} \frac{\left(\frac{\delta}{\ell}\right)^{2}}{2+\frac{3}{8} \psi^{3}\left(\frac{\delta}{\ell}\right)}=E^{\prime} \ell^{3} f\left(\frac{\delta}{\ell}\right)$. . .

where $f$ is a nondimensional function.

For other geometries of microcracks and repetitive cells, one can expect similar results. but with different expressions for functions $\phi . \psi$, and $f$.

Now, we need to carry our homogenization by macroscopic continuum Let $\epsilon$ be the (local) macroscopic strain (normal strain in the direction of principal stress $\sigma)$, and $\langle\epsilon\rangle$ the average (nonlocal) macroscopic strain, defined as $\langle\epsilon(\mathbf{x})\rangle=\ell^{-3} \int_{v_{t i}} \epsilon(\mathbf{s}) d V(\mathbf{s})$ where operator \langle\rangle denotes the spatial (nonlocal) averaging operator. $\mathbf{x}$ is the coordinate vector of the center of the cell, $s$ represents the coordinate vectors of the points of the cell, and $V(\mathbf{x})$ is the volume of the cell centered at $\mathbf{x}$. For the sake of brevity, we will delete in the following the coordinates $\mathbf{x}$ and $\mathbf{s}$, simply writing

$\langle\epsilon\rangle=\frac{1}{\ell^{3}} \int_{V} \epsilon d V$.

As one homogenizing cundition, strains $\epsilon$ must be compatible with dis- 
placement $\delta$ resulting from the crack. This is satisfied by $\delta=t\langle\epsilon\rangle$, from which

$\langle\epsilon\rangle=\frac{\delta}{t}$

The energy released from a unit cell by crack formation at constant $\delta$ can now be rewritten as

$$
W_{t}^{\prime}=E^{\prime} \ell^{3} \frac{\langle\epsilon\rangle^{2}}{2+\frac{3}{8} \psi^{3}(\langle\epsilon\rangle)}=E^{\prime} f^{3} f(\langle\epsilon\rangle)
$$

or, more precisely, $\left.W_{f}(\mathbf{x})=E^{\prime} f f \mid\langle\epsilon(x)\rangle\right]$. This equation, which shows that $W_{\text {, }}$ is a function of the average (nonlocal) strain rather than the (local) strain, is the key for the nonlocal character of continuum damage.

Note that simplifying Eq. 13 as a local relation $W_{f}(x)=E^{\prime} \ell^{3} f[\epsilon(x)]$ can make a large difference if the strain distribution approaches Dirac delta func tion, as shown in Fig. $I(d)$. This has typically happened in local continuum damage formulations and has marred finite element calculations. In fact, the nonlocality is what enforces smooth (nonlocalized) strain distributions.

Consider now that the standard stress-strain relation of continuum damage mechanics (Kachanov 1958; Lemaitre 1985; Lemaitre and Chaboche 1985). Its simplest form is

$\sigma=(1-\Omega) E^{\prime} \epsilon$

where $\Omega$ is called the damage, supposed here to be a scalar. for the sake of simplicity. Continuum damage mechanics assumes the unloading stiffness to be given by the scant modulus, which is equal to $(1-\Omega) E^{\prime}$ and corresponds to line $\overline{02}$ in Fig. 3. The density of the energy release (dissipation) resulting from damage is given by the area of triangle 0120 in Fig. 3, which is $\Omega E^{\prime} \epsilon^{2} / 2$.

According to Eq. 14, one possible definition of damage is $\Omega=1-\sigma /$ $\sigma_{e}$, where $\sigma_{c}=E^{\prime} \epsilon=$ elastic stress. Another possible definition, which we prefer to adopt here because it will lead to a simpler result, is

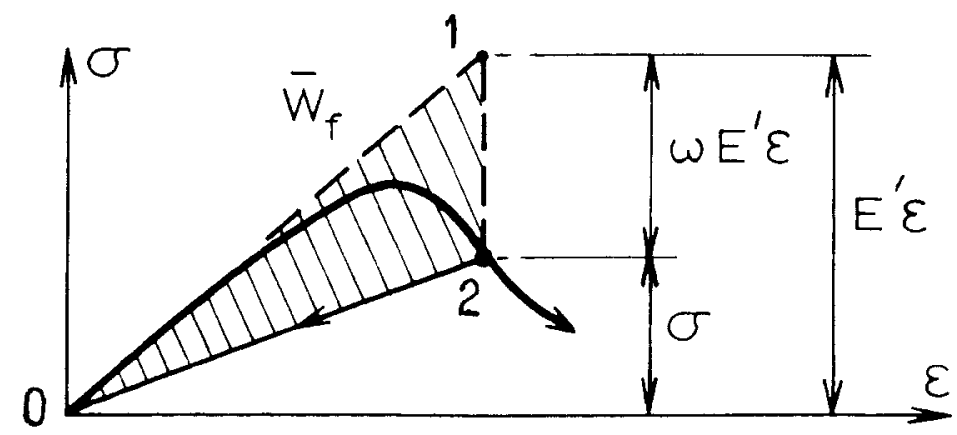

FIG. 3. Stress-Strain Relation of Continuum Damage Mechanics with Strain-Softening and Energy Release
$\Omega=\frac{\bar{W}_{f}}{\bar{W}_{c}}$

where $\bar{W}_{e}=E^{\prime} \epsilon^{2} / 2=$ elastic energy density without damage, and $\bar{W},=$ $\Omega E^{\prime} \epsilon^{2} / 2$ = density of the energy release resulting from damage.

As the second homogenizing condition, we could require: (1) The virtual work of stresses on strain variations to be the same for the unit cell and the corresponding volume of the homogenizing continuum; or (2) the encrgy release from volume $V$ of the homogenizing continuum to match the energy release $W$, from volume $V$ of the cell; or (3) the ratio in Eq. 15 to match the ratio of the energy release $W$, from the cell to the elastic strain energy $W$ of the same cell when there is no crack, i.c., to $W=t^{3} E^{\prime \prime}(\epsilon)^{2} / 2(\mathrm{ac}$ cording to Eq. 13). Although the first condition was used in Bažant (1987), here we choose the third condition, because it yields the simplest result Thus, the homogenizing condition is

$\frac{\ddot{W}_{i}}{\bar{W}_{i}}=\frac{W_{i}}{W_{e}}$

Consequently, according to Eys. 13 and 15

$\Omega=\frac{2 f(\langle\epsilon\rangle)}{\langle\epsilon\rangle^{2}}=\frac{1}{1+\frac{3}{16} \psi^{3}(\langle\epsilon\rangle)}=F(\langle\epsilon\rangle)$

where $F$ is the function defined by Eq. 17. This result shows that damage ought to be calculated from the average (nonlocal) strains. So damage caused by microcracking is nonlocal.

\section{Generalizations and Ramifications of Argument I}

The foregoing analysis simplified the response in the principal stress direction (normal to the microcrack) as uniaxial, with normal strains in the directions parallel to the crack plane being constant during crack growth. This restriction could, of course, be relaxed, but at the cost of complicating the analysis. Furthermore, in a triaxial continuum damage formulation the stress-strain relation can be written as

$\boldsymbol{\sigma}=(1-\Omega) \mathbf{E}: \mathbf{\epsilon}$

where $\boldsymbol{\sigma}$ and $\boldsymbol{E}$ are the stress and strain tensors. $\mathbf{E}$ is the fourth-order tensor of elastic moduli of undamaged material, and the damage is approximately treated as a scalar (Mazars 1984: Mazars and Pijaudier-Cabot 1989). Then, Eq. 17 can be generalized as

$\Omega=F(\langle\boldsymbol{\epsilon}\rangle)$

where $\langle\boldsymbol{\epsilon}\rangle$ is the tensor of average strains and $F$ is a scalar (invariant) function of a tensor.

A general triaxial formulation of continuum damage, such as that obtained from the microplane model, must consider damage as a fourth-order tensor, $\omega$, in which case

$\boldsymbol{\sigma}=(\mathbf{I}-\mathbf{\Omega}): \mathbf{E}: \mathbf{\epsilon}$ 
where I is the unit fourth-order tensor. In this case, the proper generalization of Eq. 17 has the form

\section{$\Omega-\mathbf{F}(\langle\epsilon\rangle)$}

where $\mathbf{F}$ is a fourth-order tensorial function of a second-order tensor

An alternative to the damage concept, which is particularly suitable for generalization to a continuum with simultaneous damage and plastic strain (Bažant and Kim 1979), is the stress-strain relation

$\boldsymbol{\sigma}=\mathbf{E}: \mathbf{\epsilon}-\boldsymbol{\sigma}^{\prime \prime}$

This is equivalent to Eq. 20 if one sets $\boldsymbol{\sigma}^{\prime r}=\boldsymbol{\Omega}: \mathbf{E}: \boldsymbol{\epsilon}$. although $\boldsymbol{\sigma}^{\prime \prime}$ can be calculated in a more general way from $\boldsymbol{\epsilon}$ and possibly also $\boldsymbol{\sigma}$. In doing this it is proper to assume that

$\boldsymbol{\sigma}^{f r}=\tilde{F}(\langle\boldsymbol{\epsilon}))$

where $\tilde{\mathbf{F}}$ is a tensorial function of a tensor. Eq. 22 means that the fracturing stress tensor ought to be considered nonlocal.

The mesh of microcrack cells has been considered in our analysis as regular and the size as constant. In reality, the mesh is irregular and the cell size, now renamed as $l$, is a random variable with a certain distribution and the average $\ell$. The nonlocal average $\langle\epsilon\rangle$ can then be defined as the statistical average of all the spatial averages of $\epsilon$ calculated for all the cell sizes $l$. By this consideration, one concludes that the nonlocal spatial averages can, in general, be defined as follows

$$
\langle\boldsymbol{\epsilon}(\mathbf{x})\rangle=\frac{1}{V_{r}} \int_{V_{r}} \alpha(\mathbf{x}-\mathbf{s}) \boldsymbol{\epsilon}(\mathbf{s}) d V(\mathbf{s})
$$

where $V_{r}$, called the representative volume (of size $\ell$ ), is the statistical mean of the values of $l^{3}$, and $\alpha(\mathbf{x}-\mathbf{s})$ is the weight function that ensues from statistical averaging over all the values of $l$. Eqs. 11-24 could now be generalized using the averaging operator 〈 > defined by Eq. 24 .

It should be noted that the averaged strain $\langle\boldsymbol{\epsilon}\rangle$ behaves as a tensor only if one considers coordinate rotations that are the same at all the points $s$ of the averaging domain. Eqs. 19, 21, and 23 were written under this tacit assumption. In general, when the coordinate rotations are not the same at all points $\mathbf{S}$, the averaged strain is not a tensor. The reason that the strain $\mathbf{\epsilon}$ must be a tensor is that its work product with the stress tensor must be a scalar. However, $\langle\boldsymbol{\epsilon}\rangle$ is not used here as a kinematic variable in a work expression; rather, it is used solely as a parameter for calculating damage, and for that purpose the fact that $\langle\boldsymbol{\epsilon}\rangle$ is not a tensor in general causes no problem. Of course, when strain invariants are used, they should be calculated, strictly speaking, as the spatial average of strain invariants rather than the invariants of the spatially averaged strain. In the case of different coordinate rotations at different points, the functional relations in Eqs. 19,21, and 23 should be reinterpreted in this sense, although for practical purposes this point seems insignificant.

In application to heterogeneous composites. such as concrete or fiber composites, the present micromechanics model consisting of microcracks within a homogencous matrix ignores the differences in the elastic moduli between the aggregate pieces and mortar. Unrealistic though this might scem, it is nevertheless possible to take the heterogeneity approximately into account by determining a proper $R$-curve $K_{R}(a)$ for the microcracks. One can find equivalent geometry-dependent $R$-curves for microcracks in a homogeneous (homogenized) elastic solid, such that they propagate in the same manner as the microcracks in the matrix of the actual elastic composite with stiffer inclusions, governed by a constant fracture toughness $(K,=$ constant $)$; Pijaudier-Cabot and Bažant (1990). This has been the main reason for considering an $R$-curve instead of a constant fracture toughness for the microcracks.

Another important simplification in argument I has been the neglect of interactions among proximate growing microcracks, which needs to be taken into account when $a / t$ is not negligible. One important question is the stability of simultaneous growth of many interacting cracks. Recent studies of path bifurcations and stability of crack systems (Bažant and Tabbara 1990; Bažant 1988) have shown that interaction results in only one crack in a homogeneous elastic solid, the others being arrested. But this is contrary to laboratory observations. It appears that the property that makes it possible for simultaneous growth of adjacent interacting cracks is the presence of harder inclusions (or perhaps also weak interfaces) in the matrix (PijaudierCabot and Bažant 1990). Their effect can be approximately described by postulating variable fracture toughness, i.e., $R$-curves, for the interacting cracks. Indeed, $R$-curve behavior of cracks has been shown to have a stabilizing effect, making simultaneous growth of adjacent cracks stable. It was for this reason that $R$-curve behavior has been included in the present formulation (Eq. 7)

We now leave the question of stability and path bifurcation aside and examine the consequence of crack interactions for nonlocality of the homogenizing continuum

\section{Argument II. Interactions among Microcracks}

Consider now an elastic body with an arbitrary system of mode-I cracks (microcracks) numbered as $\mu=1,2, \ldots, n$ [Fig. $4(a)$ ]. The solution can be calculated as a superposition of $n+1$ problems of elasticity, as shown in Figs. 4(b)-(e). We imagine that load $P$ is first applied. assuming that all the cracks remain closed as if glued |Fig. $4(b) \mid$, and we calculate the resultant $R_{\mu}=\int_{i_{\mu}} \sigma_{n} d A_{\mu}$ (positive if tensile) of the normal stresses $\sigma_{n}=\mathbf{n}_{\mu} \cdot \boldsymbol{\sigma} \cdot \mathbf{n}_{\mu}$ that are transmitted across each glued crack. $A_{\mu}$ is the area of crack $\mu, \mathbf{n}_{\mu}$ is its unit normal vector, $\boldsymbol{\sigma}$ is the stress tensor on the crack plane, and the dot denotes a singly contracted product of vectors or tensors. Subsequently, the cracks are released (or unglued), i.c., the cracks are allowed to form. This is equivalent to applying on the crack faces pairs of opposite forces $F_{\mu}$ $=\int_{A_{\mu}} \mathbf{n}_{\mu} \cdot \boldsymbol{\sigma} \cdot \mathbf{n}_{\mu} d A_{\mu}$. which are equal to $R_{\mu}$ but their positive direction is opposite to that of $R_{\mu}$ (i.e., it is such that the crack is opened by the force and the material at the crack face is compressed). To determine the effect of forces $F_{\mu}$, it is convenient to consider $n$ loading cases /Figs. $4(c),(d)$, $(e)]$, in which each of the forces $F_{\mu}$ is applied separately, and then we superpose the solutions for all these cases.

We want to determine the opening displacement $\delta_{f \mu}$ of crack $\mu$ (positive if the crack opens) caused by the given load $P$. Obviously, it is the sum of the displacements $\delta_{t \mu \nu}$ caused at crack $\mu$ by load $F_{1}$, applied at every crack 

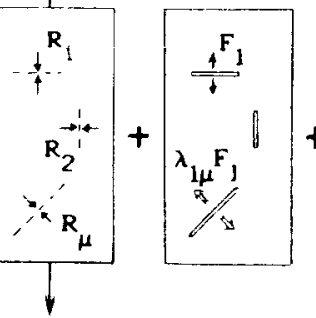

(e)

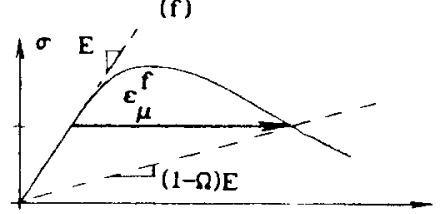

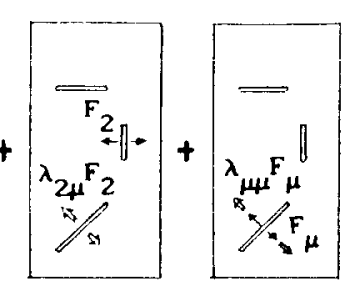

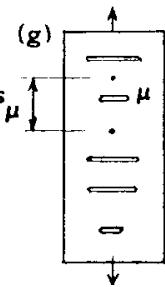

FIG. 4. Elastic Body with Interacting Cracks and Analysis of Deformations due to Cracks by Superposition of Problems with Loads Applied at Crack Faces

$v$ [i.e., the sum of the cases in Figs. $4(c),(d),(e)]$. For this purpose, it is useful to define the influence coefficients $\lambda_{\mu v}$, each of which represents the opening displacement of crack $\mu$ caused by a unit normal force $F_{v}=1$ applied on the faces of crack $v$ (the direction of this unit force is such that it causes crack $v$ to open). These coefficients are similar to those introduced by Kachanov (1987) in his analysis of stress intensity factors of crack sys tems. From Kachanov's work it is also known that the precise distribution of the crack surface tractions through which the load $F_{y}$ is applied, is unimportant and can be considered to be uniform over each crack, except when the cracks are extremely close. The crack opening displacement $\delta_{f \mu}$ [to be distinguished from the crack-tip opening displacement, (CTOD)] is understood to have a similar meaning to that in Eq. 4. It represents a certain average relative displacement of the opposite crack faces caused by the crack opening, which is work-conjugate to force $F_{\mu}$ and represents the derivative of the energy release with respect to $F_{\mu}$. With the foregoing notations, we have

$\delta_{f \mu}=\sum_{\nu=1}^{n} \lambda_{\mu \nu} F_{v}$

Here, we can substitute $F_{v}=A_{v} \boldsymbol{\sigma}_{n v}=A_{v} \mathbf{n}_{v} \cdot \boldsymbol{\sigma}_{v} \cdot \mathbf{n}_{w}$, where $\boldsymbol{\sigma}_{n v}$, $\boldsymbol{\sigma}_{v}$ are the average normal stress and the stress tensor on the crack number $v$ in its initial closed (glued) state, which can be approximated by their values at the center of a glued crack. Furthermore, we may set $A_{v}=\omega_{v} A_{y}$ where $A_{M}$ is a con stant, loosely understood as the maximum possible microcrack area at which the microcrack would join with the neighboring crack to form a continuous macrocrack (the precise meaning of $A_{M}$ is difficult to define, but for present purposes it is important only that $A_{u}$ is a constant); $\omega_{v}$ is the (local) damage in the sense of the classical geometric definition of Kachanov (1958), representing the ratio of the damaged (in this case cracked) cross-sectional area

to the total cross-sectional area of a small material element. Eq. 25 thus becomes

$\delta_{i v}=A_{u} \sum_{v=1}^{n} \lambda_{\mu \nu} \omega_{v} \mathbf{n}_{v} \cdot \mathbf{\sigma}_{v} \cdot \mathbf{n}_{v}$

This displacement has an analogous meaning to Eq. 4 and, like Eq. 4 can be regarded as a measure of the damage of the material. We now try to relate it to the damage variable $\Omega$, which appears in Eq. 18 and is as sumed, for the sake of simplicity, to be a scalar. To this end, Eq. 18 can be inverted, i.e., $\boldsymbol{\epsilon}=\mathbf{C}: \boldsymbol{\sigma} /(\mathbf{1}-\Omega)$, where $\mathbf{C}$ is the fourth-order compliance tensor of the uncracked elastic material, whose $6 \times 6$ matrix $C$ is the inverse of the matrix $\mathbf{E}$ of the elastic moduli tensor $\mathbf{E}$, that is $\mathbf{C}=\mathbf{E}^{-1}$. The normal strain of the macroscopic continuum (with smeared cracks) at the location of the center of crack $\mu$ and in the direction normal to crack $\mu$ is $\epsilon_{n \mu}=$ $\mathbf{n}_{\mu} \cdot \boldsymbol{\epsilon}_{\mu} \cdot \mathbf{n}_{\mu}$ or

$\epsilon_{n \mu}=\frac{1}{1-\Omega} \epsilon_{n \mu}^{e}$

$\boldsymbol{\epsilon}_{n \mu}^{e}=\mathbf{n}_{\mu} \cdot \mathbf{C}: \boldsymbol{\sigma}_{\mu} \cdot \mathbf{n}_{\mu}$

where $\epsilon_{n \mu}^{\rho}$ is the elastic normal strain of the uncracked material in the direction normal to crack $\mu$.

The normal fracturing strain $\epsilon_{n \mu}^{f}$ caused by smearing the relative displacements $\delta_{f \mu}$ of direction $n$ resulting from fracture can be written as $\delta_{f \mu} / s$, where $s$ is a constant, loosely understood as the average spacing of the microcracks of this orientation (again, the precise meaning of $s$ is difficul to define but for our purposes it is important only that $s$ is a constant). In view of our assumption of scalar damage, we must assume the displacements $\delta_{f \mu}$ occur with equal frequency in all spatial directions. The displacements in the directions normal to $\mathbf{n}$ do not contribute to the strain in direction $\mathbf{n}$. One gets $\epsilon_{n \mu}=k_{1} \delta_{f \mu} / \mathrm{s}$, where $k_{1}$ is a constant determined by integration over all strain directions. Adding to this the elastic normal strain of the uncracked material in the same direction, we get

$\epsilon_{n \mu}=\epsilon_{n \mu}^{c}+\epsilon_{n \mu}^{\prime}$

$\epsilon_{n \mu}^{f}=k_{1} \frac{\delta_{f \mu}}{s}$

Equating the right-hand sides of Eqs. 27 and 28 and solving the resulting equation for macroscopic scalar continuum damage $\Omega$ at the location of crack $\mu$, we obtain, after rearrangements

$\Omega_{\mu}=\left(1+\frac{1}{\sum_{\nu} \phi_{\mu \nu} \omega_{\nu}}\right)$

where

$\phi_{\mu v}=k_{1} \frac{A_{M}}{s} \frac{\mathbf{n}_{v} \cdot \boldsymbol{\sigma}_{v} \cdot \mathbf{n}_{v}}{\mathbf{n}_{\mu} \cdot \mathbf{C}: \boldsymbol{\sigma}_{\mu} \cdot \mathbf{n}_{\mu}} \lambda_{\mu v}$ 
The fact that Eq. 29 involves a sum over the values of $\omega_{v}$ at the locations of all the microcracks proves again that continuum damage $\Omega$ is a nonlocal variable. This property is a consequence of crack interactions characterized by influence coefficients $\lambda_{\mu \nu}$ of the crack system. Because the influence coef ficients are negligibly small for all those pairs of cracks that are not sufficiently close to one another, the summation in Eq. 29 is effectively carried out only over a certain finite neighborhood of the point.

The fact that the weight coefficients $\phi_{\mu}$ of the sum in Eq. 29 involve the stresses at various points is a complicating feature, compared with the av eraging rule in Eqs. 17 or 24 , as well as the recent practice of nonlocal finite element analysis. It means that the weight function for nonlocal spatial integration is not a fixed material property, but a material function varying with the stress tensor field (it might be, however, that this complicating feature could be ignored for practical purposes).

However, in the special case of a uniform hydrostatic (volumetric) stress field, the stresses disappear from the coefficients of the summation and Eq. 30 reduces to

$\phi_{\mu v}=\frac{k_{1} A_{M}}{C s} \lambda_{\mu v}$

where $C$ is the elastic volumetric (bulk) compliance of the material

When the local damage values $\omega_{\mu}$ are sufficiently small, Eq. 29 can bo approximated as

$\Omega_{\mu}=\sum_{i=1}^{n} \phi_{\mu \nu} \omega_{\nu}$

Coefficients $\phi_{\mu \text {. }}$ have the meaning of discrete values of the weight function for nonlocal (spatial) integration. (Note that, in contrast to the previous formulations, the weights $\phi_{\mu \nu}$ are not, in general, normalized, that is, $\Sigma \phi_{\mu \nu}$ is gencrally not equal to 1 .)

In the special case of hydrostatic stress o (Eq. 31), the influence coefficients of the crack system play directly the role of the discrete values of the weight function for nonlocal spatial integration. [This idea has previously been suggested, without proof, in general terms in Pijaudier-Cabot and Bažant (1990) and Pijaudier-Cabot and Berthaud (1990).l

According to Eqs. 29-32, the weight function for spatial integration or summation is not a fixed material property, but depends on the current sizes and configurations of the microcracks, because the influence coefficients $\lambda_{\mu \nu}$ depend on them. Thus, as the microcrack system evolves with the progress of loading, the weight function evolves with it. (Whether and when this dependence can be neglected in practical computation, as done so far, remains to be checked.)

If the system of microcracks is statistically homogeneous in space (as can often be the case for the initial state of the material), the influence coefficients $\lambda_{\mu l}$, thus also the weight function, are independent of the macroscopic spatial coordinates. Even then, however, the weight function is affected by the presence of the boundary of the body and depends on the boundary shape because $\lambda_{\mu \nu}$ exhibit such dependence.

To recapitulate the basic idea of the foregoing analysis, the local damage can be defined as the area fraction occupied by the crack (or cracks) in a small material element, and the nonlocal damage, which enters the stressstrain relation, can be defined as a variable proportional to the crack opening displacements. Because of these different physical meanings of the local damage and nonlocal damage, the nonlocal spatial integral for, in the discrete version, the sum) does not have the meaning of an averaging operation, that is, the weights $\phi_{\mu v}$ are not normalized, in general.

The fact that $\omega$ is not an adequate measure of damage to be used in the stress-strain relation is clear from the observation that a pair of two small microcracks gives the same value of $\omega$ as one larger microcrack, an area of which is equal to the combined area of the two small microcracks. Yet, the actual damage to the material from the larger microcrack must be much greater, because the crack opening displacement is proportional to the cube of crack diameter (Eq. 4) in the case of circular cracks.

An important result to be noted from Eqs. 30-32 is that the variable that is nonlocal (i.e., the variable subjected to spatial summation or, in the continuum approximation, spatial integration) is solely the damage, as in argument $\mathrm{I}$. The stress, elastic strain, and total strain remain local.

In contrast to argument $\mathrm{I}$, the foregoing analysis did not take into account the conditions of crack propagation and ignored the fracture energy $G_{f}$ or the $R$-curve of the material. But that is not a detriment as far as the calculation of damage is concerned. The value of damage $\Omega$ determines the current secant stiffness [Fig. $4(f)$ ], and so do the current crack lengths. The growth of cracks, for which the value of fracture energy (or the $R$-curve) does have to be considered, determines the tangent stiffness. The foregoing analysis would have to be extended by a crack growth law in order to furnish the growth law of $\Omega$ and the tangent stiffness ffor a general approach to this see Bažant and Tabbara (1990)].

\section{Special Case and Generalization of Argument II}

Consider now the special case of uniaxial stress $\sigma$ of direction $x$, with uniaxial damage $\omega$. All the microcracks are, in this case, normal to $x$ [Fig. 4(g)]. We have $F_{\mu}=A_{\mu} \sigma_{\mu}, A_{\mu}=\omega_{\mu} A_{M}$. We can now assume the microcrack spacing to be variable, in general, denoting by $s_{\mu}$ the distance between the midpoints between the adjacent cracks [Fig. 4(g)]. Then $\epsilon_{\mu}^{f}=\delta_{f \mu} / s_{\mu}$. Denoting by $\Omega_{\mu}$ the damage at crack $\mu$ that enters the stress-strain relation, the condition of equivalence to continuum damage mechanics requires that $\left(\sigma_{\mu}\right)$ $\left.E^{\prime}\right)+\epsilon_{\mu}^{\prime}=\sigma_{\mu} /\left(1-\Omega_{\mu}\right) E^{\prime}$. From these equations, one gets Eq. 25 again, in which

$\phi_{\mu \nu}=c_{0} \frac{\sigma_{v}}{\sigma_{\mu}} \lambda_{\mu \nu}$

$c_{0}=\frac{E^{\prime} A_{M}}{s_{\mu}}$

$\left(c_{0}=\right.$ constant $)$. If the thickness of the uniaxially stressed bar is constant, then $\sigma_{\mu}=\sigma_{v}$ and $\phi_{\mu \nu}=c_{0} \lambda_{\mu \nu}$.

The assumption of scalar damage, although frequently made for the sake of simplicity, is of course a gross simplification. Consider now general loading and general anisotropic nonlocal damage, which is a fourth-order tensor, 
$\boldsymbol{\Omega}$. The normal force components canceling the crack openings (glued cracks) are $F_{v}=A_{v} \mathbf{n}_{v} \cdot \boldsymbol{\sigma}_{v} \cdot \mathbf{n}_{v}$, where $A_{v}=\omega_{v} A_{m}$, as before. The normal relative displacement of crack $\mu$ is $\delta_{f \mu}=\Sigma_{\nu} \lambda_{\mu \nu} F_{\nu}$. Now the macro-stress tensor at location $\mu$ is

$\sigma_{\mu}=\mathbf{E}:\left(\boldsymbol{\epsilon}_{\mu}-\boldsymbol{\epsilon}_{\mu}^{\prime}\right)$

with

$\boldsymbol{\epsilon}_{\mu}^{\prime}=\underline{\mathbf{n}_{\mu} \otimes \mathbf{n}_{\mu} \delta_{j \mu}}$

where $\mathbf{E}$ is the fourth-order tensor of clastic moduli of the uncracked material; $\epsilon_{\mu}^{\prime}$ is the (nonlocal) tensor of fracturing strain caused by $\delta_{j \mu}$, which is obviously nonlocal because it involves a spatial summation; $s_{\mu}$ is a constant approximately characterizing the crack spacing at $\mu$; and symbol $\otimes$ denotes the tensor (in this case, dyadic) product, which is contracted on no index. Substituting the previous expressions for $\delta_{f_{\mu}}$ and $F_{\mu}$, we obtain for the (nonlocal) fracturing strain tensor the expression

$\boldsymbol{\epsilon}_{\mu}^{\prime}=\mathbf{n}_{\mu} \otimes \mathbf{n}_{\mu} \frac{A_{M}}{s_{\mu}} \sum_{v=1}^{n} \Lambda_{\mu v} \omega_{v}\left(\mathbf{n}_{v} \cdot \boldsymbol{\sigma}_{v} \cdot \mathbf{n}_{v}\right)$

Now, we need to relate this to the damage formulation, which, in general, has the form

$\boldsymbol{\sigma}_{\mu}=\mathbf{R}_{\mu}: \boldsymbol{\sigma}_{\mu}^{e}$

$\boldsymbol{\sigma}_{\mu}^{e}=\mathbf{E}_{\mu}: \epsilon_{\mu}$

$\mathbf{R}_{\mu}=\mathbf{I}-\boldsymbol{\Omega}$

where $\boldsymbol{\sigma}_{\mu}^{e}$ is the elastic stress tensor in the uncracked solid at location $\mu, \mathbf{I}$ is the fourth-order unit tensor, and $\mathbf{R}_{\mu}$ is the fourth-order tensor of integrity (nondamage)

Nonlocal damage $\Omega$ must now be such that Eq. 36 is equivalent to Eqs. 34-35. which means solving the equation $\boldsymbol{\sigma}_{k}=\mathbf{R}_{\mu}: \boldsymbol{\sigma}^{i}$ (or $\sigma_{\mu i}=$ $R_{i j k m} \sigma_{\mu k m}^{\prime \prime}$ in the Cartesian component form). It turns out, however, that this condition. which represents a set of six algebraic lincar equations, is insufficient to determine tensor $\Omega$, which has 21 independent components. There are infinitely many solutions, for example

$\mathbf{R}_{\mu}=\frac{\boldsymbol{\sigma}_{\mu} \otimes \boldsymbol{\sigma}_{\mu}}{\boldsymbol{\sigma}_{\mu}: \boldsymbol{\sigma}_{\mu}^{e}}$

or

$\boldsymbol{\sigma}_{\mu} Q \boldsymbol{\sigma}_{\mu}^{\prime}$

$\boldsymbol{\sigma}_{\mu}^{\prime}: \boldsymbol{\sigma}_{\mu}^{\prime \prime}$

$\boldsymbol{\sigma}_{\mu} \otimes \boldsymbol{\sigma}_{\mu} \cdot \boldsymbol{\sigma}_{\mu}$

$\operatorname{tr}\left(\boldsymbol{\sigma}_{\mu} \cdot \boldsymbol{\sigma}_{\mu} \cdot \boldsymbol{\sigma}_{\mu}^{\prime}\right)$

The general fourth-order damage tensor can be determined only if the crack growth law and the tangential stiffness tensor are known and the evolution of $\mathbf{R}_{\mu}$ or $\boldsymbol{\Omega}_{\mu}$ is traced incrementally from the initial state. But the secondorder fracturing strain tensor can be determined from the current state alone, as we have seen.

\section{THIRD ARgumENT: INHOMOGENEITIES}

Consider a group of hard aggregate pices of concrete embedded in a relatively soft matrix (mortar), as shown in Fig. 5. Obviously. the formation of the microcrack shown will not be determined by the local strain in the crack center. Rather, it will be determined by the overall deformation of the group of aggregates, particularly the relative displacement of the adjacent aggregate pieces. This deformation is characterized by the averaged strain from the corresponding domain (representative volume) of the smoothing continuum (the circle shown), rather than the local strain at the center of this domain.

This argument (Bažant 1986), however, defies simple analytical treatment. For this reason, the effect of inhomogeneities has been studied numerically (Bažant et al. 1990). Concrete specimens were approximately simulated as a randomly generated configuration of aggregate pieces with a prescribed size distribution. The pieces were assumed to interact only by central forces whose law was characterized by prescribed strength and postpeak softening of a slope that corresponds to a specified interparticle fracture energy. The argument for the nonlocality of damage emerged indirectly, through the size effect. It was found that the values of the nominal strengths of geometrically similar specimens of different sizes agree quite well with the size effect law of the same type exhibited by finite element solutions with nonlocal damage or some other localization limiter (as well as by test results); the usual (local) finite element solutions exhibit no size effect (in the deterministic approach) This provides another argument for the nonlocal approach in some of its various possible variants.

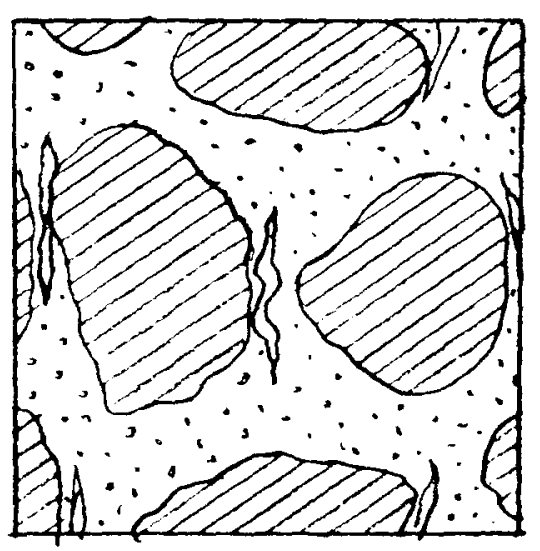

FIG. 5. Cracking in Group of Inhomogeneities Embedded in Soft Matrix 


\section{CONCLUSIONS}

1. Argument I. which is based on a simplified micromechanics analysis of smali noninteracting cracks arranged in a cubic lattice, shows that one reason for continum damage resulting from microcracking to be nonlocal is that the fracturing strain caused by damage is the result of the release of stored energy from a microcrack neighborhood the size of which is not negligible.

2. Argument II, in which local damage is defined (geometrically) as the damaged (cracked) area fraction and the nonlocal damage is defined (mechanically) as a variable proportional to the microcrack opening displacements, shows that the existence of interactions among microcracks implies nonlocality.

3. Argument II indicates that the local damage and nonlocal damage do not have analogous physical meanings, and consequently the nonlocal spatial integral (or summation) does not have the meaning of averaging

4. According to argument II, the weight function for nonlocal spatial integration evolves as a function of the sizes and configuration of the microcracks and is affected by the proximity of the boundary of the body and the boundary shape.

5. Argument II indicates that the weight function for nonlocal spatial inte gration is a fixed material property, independent of the stresses, only if the stress state is hydrostatic and the damage is sufficiently small. Otherwise, the weigh function depends on the stress tensor field in a certain neighborhood of the point.

6. Both arguments indicate that the only state variable that is nonlocal is the damage (or the fracturing strain). The stress, elastic strain, and total strain (as a kinematic variable associated by work with the stress) are local.

7. A third argument for the nonlocal approach is indirectly provided by the properties of numerical simulations of specimens with microstructural inhomogeneities, simulated as random particle systems characterized by interparticle force displacement law with postpeak softening.

\section{Remark}

The dependence of the weight function on the stresses and the current state of damage has been ignored in practical calculations so far, yet often the agreement with test results, particularly those on the size effect, has been satisfactory (e.g., Bažant and Ožbolt 1990; Ožbolt and Eligehausen 1990). Whether this simplified approach is indeed admissible, and under which conditions, is an interesting question that should be explored.

\section{ACKNOWLEDGMENT}

The paper was written during the writer's visiting appointment at Lehrstuhl für Mechanik (director, Prof. H. Lippmann), Technische Universität München, Germany, supported under Humboldt Award of Senior U.S. Scientist. Further support was received from the U.S. Air Force Office of Scientific Research under a grant to Northwestern University, and from Center for Advanced Cement-Based Materials at Northwestern University.

\section{Appendix. References}

Bažant, Z. P. (1984). "Imbricate continuum and its variational derivation." J. Engrg. Mech. ASCE, $110(2), 1693-1712$.
Bažant, Z. P. (1986). "Mechanics of distributed cracking." Appl. Mech. Rev.. ASME, $39(5), 675-705$

Bažant, Z. P. (1987). "Why continuum damage is nonlocal: justification by microcrack array." Mech. Res. Commun., 14(516), 407-419.

Bažant, Z. P., Belytschko, T. B., and Chang, T. P. (1984). "Continuum theory for strain-softening." J. Engrg. Mech.. ASCE, 110(12), 1666-1692.

Bažant, Z. P., and Kazemi, M. T. (1990). "Size effect in fracture of ceramics." J Am. Ceramic Soc, 73(7), $1841-1853$

Bažant, Z. P., and Kim. S. S. (1979), "Plastic-fracturing theory for concrete." J Engrg. Mech. ASCE, 105, 407-428

Bažant, Z. P., and Lin, F.-B. (1988). "Nonlocal yield limit degradation." Int. J. Numer. Methods Engrg., 26, 1805-1823.

Bažant, Z. P., and Lin, F.-B. (1988). "Nonlocal smeared cracking model for concrete fracture." J. Struct. Engrg., ASCE, $114(11), 2493-2510$.

Bažant, Z. P., and Ožbolt, J. (1990). "Nonlocal microplane model for fracture, damage, and size-effect in structures." J. Engrg. Mech., ASCE, 116(11), 2484-2504

Bažant, Z. P., and Pijaudier-Cabot, G. (1988). "Nonlocal continuum damage, 10 . calization instability and convergence." J. Appl. Mech.. ASME, 55, 287-293.

Bažant, Z P and Tabbara, M. R (1990) "Bifurcation and stability of structure with interactive propayating cracks." Report No.90-4/6166. Center for Advanced Cement-Based Materials, Northwestern Univ., Evanston, III.

Bažant, Z. P., Tabbara, M. R., Kazemi, M. T., and Pijaudier-Cabot, G. (1990) "Random particle model for fracture of aggregates or fiber composites." J. Engrg. Mech., ASCE, 116(8), 1686-1705.

Broek, D. (1974). Elementary engineering fracture mechanics. Sijthoff and Noor dhoff International Publishers, Netherlands

Dempsey, J. (1990). ASCE Materials Engineering Congress held in Denver, in press. Eringen, A. C (1966). "A unified theory of thermomechanical materials, Int $J$. of Engrg. Science, 4, 179-202.

Eringen, A. C. (1965). "Theory of micropolar continuum." Proc., Ninth Midwestern Mechanics Conference, Univ of Wisconsin, Madison, Wis., 23-40.

Eringen, A. C. and Ari, N. (1983). "Nonlocal stress field at Griffith crack." Crist. Latt. and Amorph. Mater., 10, 33-38.

Eringen, A. C., and Edelen, D. G. B. (1972). "On nonlocal elasticity." Int. J. Engrg. Sci., $10,233-248$

Kachanov, L. M (1987). "Elastic solids with many cracks-a simple method of analysis." Int. J. Sol. Struct., 23(1), 23-43.

Kachanov, L. M. (1958). "Time of rupture process under creep conditions." Lzvestia Akademii Nauk, the Soviet Union, 8, 26-31 (in Russian).

Knott, J. F. (1973). Fundamentals of fracture mechanics. Butterworth, London, U.K

Kröner, E. (1968). "Elasticity theory of materials with long-range cohesive forces." Int. J. Sol. Struct., 3, 731-742.

Krumhansl, J. A. (1968). "Some considerations of the relations between solid state physics and generalized continuum mechanics." Mechanics of generalized continua, E. Kröner, ed., Springer-Verlag, Heidelberg, Germany, 298-331.

Kunin, I. A. (1968). "The theory of elastic media with microstructure and the theory of dislocations." Mechanics of generalized continua, E. Kröner, ed., SpringerVerlag, Heidelberg, Germany, 321-328

Lemaitre, J. (1985). "A continuous damage mechanics model for ductile fracture." J. Engrg. Mater. Tech., 107, 83-89.

Lemaitre, J., and Chaboche, J. L. (1985). "Mécanique des matériaux solides, DunodBordas, Paris, France (in French).

Levin, KV. M. (1971). "The relation between mathematical expectation of stress and strain tensors in elastic microheterogeneous media." Prikl. Mat Mekh., 35, $694-701$.

"Stress intensity factors handbook." (1987). Y Murakami, ed., Pergamon Press, Oxford, New York, NY

Mazars, J. (1984). "Application de la mecanique de l'endommagement au comportement non-imearre et a ta rupture du beton de structure, these de doctorat 
d'Etat es Sciences Physiques. Universite Paris VI. France.

Mazars, J., and Pijaudier-Cabot, G. (1989). "Continuous damage theory: application to concrete." J. Engrg. Me'ch., ASCE, 115(2), 345-365.

Ozbolt, J., and Eligehausen. R. (1990). "Size effect in anchorage behavior." Proc. Fracture Behavior and Design of Materials and Structures, European Conf. on Fracture. (ECF8), Torino, Italy.

Pijaudier-Cabot, G., Bažant, Z. P., and Berthaud, Y. (1990). "Interacting crack systems in particulate or tiber reinforced composites." Proc., Fifth Int. Conf. on Numerical Methods in Fracture Mechanics, Freiburg, Germany. A. R. Luxmoore and D. R. J. Owen, eds, , 403-414.

Pijaudier-Cabot, G., and Bažant, Z. P. (1987). "Nonlocal damage theory." J. Engrg. Mech., ASCE, 113(10), 1512-1533.

P. (1990) "Propagation of interacting cracks in an clastic solid with inclusions." preliminary report, Northwestern Univ., Evanston, 11

Pijaudier-Cabot, G., and Berthaud, Y. (1990). "Effects des interactions dans l'endommagement d'un milieu fragile. Fornulation non locale." Comptes Rendus, Acad. Sci., Paris, France, 310(II), 1577-1582.

Tada, H., Paris, P. C., and Irwin, G. R. (1985). "The stress analysis of cracks handbook." Second Ed. . Paris Productions, Inc., St. Louis, Mo. 